Med Klin Intensivmed Notfmed 2021 · 116:357 https://doi.org/10.1007/s00063-021-00811-6 Online publiziert: 6. April 2021

(c) Springer Medizin Verlag GmbH, ein Teil von Springer Nature 2021

\section{Lothar Engelmann}

Leipzig, Deutschland

\title{
Ist der pH noch wichtig?
}

\section{Leserbrief $\mathbf{z u}$}

Kabbani A-R, Tergast TL, Manns MP und Maasoumy B (2021) Behandlungsstrategien des akut-auf-chronischen Leberversagens. Med Klin Intensivmed Notfmed 2021:116:3-16. https://doi.org/10.1007/s00063-019-00613-x

Verehrte Autoren,

zunächst vielen Dank für Ihre Übersichtsarbeit $\mathrm{zu}$ einem Thema, das die Medizin seit Jahrzehnten beschäftigt, zu dem zwar über diesen Zeitraum ein enormer Wissenszuwachs gelang, am Ende aber nur bescheidene Therapieerfolge resultierten, sieht man von Leberunterstützungsverfahren und der Lebertransplantation ab. Umso mehr, scheint mir, sollte jedem Hinweis nachgegangen werden, der sich auch mit symptomatischen Therapieansätzen aus der Vergangenheit beschäftigt.

Ich selbst habe 1969 zum Thema „Der Ammoniakspiegel bei Lebererkrankungen“ bei R. Emmrich in Leipzig promoviert. Dem Ammoniakspiegel kam damals schon eine diagnostische Bedeutung bei der Bewertung der hepatischen Enzephalopathie zu und seine Bestimmung erfolgt bis in die Gegenwart. In der Mehrzahl der Fälle von Leberausfall mit Enzephalopathie ist der Ammoniakspiegel erhöht. Meist bleibt es bei dieser Feststellung als Bestätigung der Enzephalopathie. Sie stellen in Ihrem Beitrag therapeutische Eingriffe an der Darmflora, diätetische Maßnahmen, die orale $\mathrm{Ga}$ be von verzweigtkettigen Aminosäuren, die intravenöse Zufuhr von L-OrnithinL-Aspartat und den Aufbau von quergestreifter Muskulatur dar, um den Ammoniakspiegel zu beeinflussen. Keine Erwähnung fand allerdings die Vermeidung von Alkalosen, durch Hypokaliämie oder Hyperventilation bedingt.

Ammoniak liegt bei normalem $\mathrm{pH} z u$ $97,5 \%$ als Ion und zu nur 2,5\% als physikalisch gelöster Ammoniak vor und nur letzterer kann Zellmembranen permeieren und zu einer hepatischen Enzephalopathie beitragen [1]. Unter Abnahme der Wasserstoffionenkonzentration ( $\mathrm{pH}$ Anstieg) ändert sich der Dissoziationsgrad derart, dass der Anteil des frei diffusiblen Ammoniaks an der Blut-HirnSchranke ansteigt und damit mehr Ammoniak in die Hirnzellen gelangt.

Ich habe in meinen Jahren in der Intensivmedizin immer darauf geachtet, bei hepatischen Enzephalopathien Alkalosen zu vermeiden bzw. zu korrigieren, und denke, damit gut gefahren zu sein.

Gelten diese Vorstellungen aus der Mitte des letzten Jahrhunderts mit ihren Konsequenzen noch immer oder sind sie obsolet?

Ihr L. Engelmann

\section{Korrespondenzadresse}

Prof. Dr. Lothar Engelmann

Sigebandweg 25, 04279 Leipzig, Deutschland pdle@gmx.de

Interessenkonflikt. L. Engelmann gibt an, dass kein Interessenkonflikt besteht.

\section{Literatur}

1. Bromberg PA, Robin ED, Forker CE (1960) The existence of ammonia in blood in vivo with observations of the significance of the $\mathrm{NH}_{3}-\mathrm{NH}_{4}{ }^{+}$system. JClin Invest 39:332 